\title{
Numerical Strategies for Stroke Optimization of Axisymmetric Microswimmers*
}

\author{
François Alouges $^{1}$, Antonio DeSimone ${ }^{2}$, and Luca Heltai ${ }^{\dagger, 2}$ \\ ${ }^{1}$ CMAP UMR 7641, École Polytechnique CNRS, , Route de \\ Saclay, 91128 Palaiseau Cedex - France \\ ${ }^{2}$ SISSA-International School for Advanced Studies, , Via \\ Bonomea 265, 34100 Trieste - Italy
}

\begin{abstract}
We propose a computational method to solve optimal swimming problems, based on the boundary integral formulation of the hydrodynamic interaction between swimmer and surrounding fluid and direct constrained minimization of the energy consumed by the swimmer.

We apply our method to axisymmetric model examples. We consider a classical model swimmer (the three-sphere swimmer of Golestanian et al.) as well as a novel axisymmetric swimmer inspired by the observation of biological micro-organisms.
\end{abstract}

\footnotetext{
${ }^{*}$ Preprint SISSA 33-2009-M.

${ }^{\dagger}$ Corresponding Author. Email: Luca Heltai <luca.heltai@sissa.it>; Tel.: +39 040-3787449; Fax: +39 040-3787528
} 


\section{Swimming at low Reynolds Numbers}

Swimming is the ability to advance within a fluid by performing cyclic shape changes, in the absence of external propulsive forces. One of the main difficulties of swimming at small scales is given by the time-reversal property of Stokes flows, which describe hydrodynamics at low Reynolds numbers.

The physical implication of this mathematical property is that simple swimming strategies used in nature at larger scales, for example by scallops, where the same shape change is executed forward and backward at different velocities to achieve propulsion in one direction, do not work for micro-, or nano-swimmers. At this scale, swimmers have to undergo nonreciprocal deformations in order to achieve propulsion. Also known as the Scallop-Theorem, this fact is discussed by Purcell in Ref. [1, where a simple mechanical device that can indeed swim at low Reynolds numbers, the threelink swimmer, is proposed. A mathematical statement of the scallop theorem and its proof can be found in Ref. [2]. More recently several other simple swimmers have been presented, for example, in Golestanian and Najafi [3] and Avron et al. [4]

A mathematical approach to the problem of finding an optimal stroke has been proposed by Alouges et al. in Ref. [5], where it is shown how to formulate and solve numerically the problem of finding optimal strokes for low Reynolds number swimmers by focusing on the three-sphere swimmer of Najafi and Golestanian [3] (a simple, yet representative example). The analysis carried out in Ref. [5] shows how to address quantitatively swimming as the problem of controlling shape in order to produce a net displacement at the end of one stroke. By casting it in the language of control theory, the problem of swimming reduces to the controllability of the system, and the search of optimal strokes to an optimal control problem leading to the computation of suitable sub-Riemannian geodesics.

The use of numerical algorithms to find optimal strokes can lead to dramatic improvements. For the three-sphere swimmer, one can achieve an increase of efficiency exceeding $300 \%$ with respect to more naive proposals. [5] The simplicity of three-sphere swimmer, which is a system with two degrees of freedom, enables one to carry out the analysis in full detail. The study of biologically relevant swimmers, however, requires more abstract mathematical tools and more efficient numerical algorithms. The aim of this paper is to introduce further numerical tools to overcome some of the computational limits of the numerical method used in Ref. [5], opening up the possibility 
to treat more and more complex swimmers such as the new model swimmer described in section 4, which is inspired by biological micro-swimmers like unicellular eukaryotic algae.

For simplicity, we focus on the special case of axisymmetric swimmers, which provides an interesting balance between complexity and generality of the attainable results. A complete theory to analyze axisymmetric swimmers whose shape depends on finitely many parameters and a general method to determine strokes of maximal efficiency is presented in Ref. 6. The main feature qualifying this approach is the possibility of resolving hydrodynamic interactions arising from the swimmer motion in their full complexity, without being confined to asymptotic regimes (dilute limits for assemblies of spheres, slender body hydrodynamics for one-dimensional objects) in which explicit formulas for hydrodynamic forces are available.

This work follows a similar line of thought. The main novelties introduced here are the use of a boundary integral formulation for the solution of the axisymmetric Stokes flow induced by the motion of the swimmer, and the resolution of the optimal control problem via direct constrained minimization of the functional giving the energy spent by the swimmer. This should be contrasted with the resolution of the hydrodynamic interactions via the finite element method that was used in Ref. [5] and Ref. [6], and the resolution of the Euler-Lagrange equation associated with the energy minimization problem via an ODE scheme coupled with a shooting method to enforce periodicity of the swimming stroke.

The accuracy that can be obtained with the new method is a few orders of magnitude better than with the previous one, due to the possibility of resolving the swimmer details at a much smaller scale at a comparable computational cost. In addition to the improved accuracy granted by the use of boundary integrals, the direct solution of the constrained minimization problem enlarges the class of optimization problems we can consider. In particular, it enables us to consider also inequality constraints on the shape variables. This is often necessary in order to restrict the set of admissible shape changes to those that are also biologically plausible.

The rest of this paper is organized as follows. In section 2 we derive the equations characterizing optimal strokes, while in section 3 we propose a strategy for their numerical solution which is alternative to the ones presented in Ref. [5] and Ref. [6]. We validate our method by comparing with previous results on the three-sphere swimmer, and by calculating optimal strokes for a new model axisymmetric swimmer in section 4 . 


\section{Governing Equations}

We consider an axisymmetric micro-organism or micro-robot swimming at low Reynolds numbers in a three dimensional fluid at rest at infinity. No interaction with other obstacles is considered.

The swimmer occupies at time $t$ the (unknown) portion of space denoted by $\Omega(t) \subset \Re^{3}$, with boundary $\partial \Omega(t)=\Gamma(t)$, while the surrounding region $\Re^{3} \backslash \Omega(t)$ is occupied by the fluid.

We are interested in finding the "optimal stroke" of period $T$, directed along the axis of symmetry of the swimmer, and that will take the swimmer from the position $\Omega_{0}$ to the position $\Omega(T)=\Omega_{0}+c e$, where $c / T$ is the average swimming velocity.

According to whether we want to optimize performance or energy consumption, different optimality criteria can be adopted. In this paper we select the minimization of the energy spent by a swimmer to swim at fixed (given) average velocity $c / T$ as the optimality criterion.

\subsection{Exterior Hydrodynamic Interactions}

Independently of the optimality criterion that one chooses, any swimmer needs to satisfy certain constraints which are required for the problem to be well posed and for it to actually represent a physically admissible swimmer.

An organism is said to be swimming if it advances while its shape changes periodically in time by following the hydrodynamics of the surrounding fluid, in the absence of external forces or torques.

More precisely, then, a low Reynolds number swimmer satisfies the following key properties:

- the velocity and force density at the surface of the swimmer are linked by the exterior Stokes equations satisfied by the surrounding fluid;

- the shape of the swimmer changes periodically in time, driving the surrounding flow;

- the force is generated internally by the swimmer, that is, the total external force and torque that act on the swimmer are zero.

On the exterior part of the swimmer, this is expressed by the following 
system of equations, satisfied for each $t$ in $[0, T]$ :

$$
\begin{array}{ll}
-\eta \Delta \boldsymbol{u}(\boldsymbol{x}, t)+\nabla p(\boldsymbol{x}, t)=-\nabla \cdot \boldsymbol{\sigma}=0 & \text { in } \Re^{3} \backslash \Omega(t) \\
\nabla \cdot \boldsymbol{u}(\boldsymbol{x}, t)=0 & \text { in } \Re^{3} \backslash \Omega(t) \\
\boldsymbol{u}(\boldsymbol{x}, t)=\boldsymbol{v}(\boldsymbol{x}, t) & \text { on } \Gamma(t) \\
\quad \lim _{|\boldsymbol{x}| \rightarrow \infty}|\boldsymbol{u}(\boldsymbol{x}, t)|=0 & \\
\lim _{|\boldsymbol{x}| \rightarrow \infty}|p(\boldsymbol{x}, t)|=0 & \\
\frac{\partial \boldsymbol{X}}{\partial t}=\boldsymbol{v}(\boldsymbol{X}, t) & \\
\Gamma(T)=\boldsymbol{R} \Gamma(0)+\int_{0}^{T} \int_{\Gamma} \boldsymbol{v} \mathrm{d} \Gamma \mathrm{d} t=\boldsymbol{R} \Gamma(0)+c \boldsymbol{e} & \\
\int_{\Gamma(t)} \boldsymbol{\sigma n}=0 & \\
\int_{\Gamma(t)} \boldsymbol{x} \wedge \boldsymbol{\sigma n}=0 &
\end{array}
$$

where $\boldsymbol{u}$ and $p$ are the velocity and hydrodynamic pressure fields in the exterior domain $\Re^{3} \backslash \Omega(t), \eta$ is the viscosity of the fluid, $\boldsymbol{v}$ is the velocity at the surface of the swimmer, $\boldsymbol{X}$ is a material point on the surface $\Gamma(t)$ and $\boldsymbol{e}$ is the direction along which the swimmer is moving.

The set of Equations (1a) describes the conservation of momentum and mass in the Stokes fluid surrounding the swimmer, with zero boundary conditions at infinity.

In Eq. (1b) we grouped the no-slip boundary condition (each material particle of the surface of the swimmer is following the evolution of the flow, i.e., no slip occurs between the fluid and the swimmers) and the periodicity of the swimmer shape, where $c:=\int_{0}^{T} \boldsymbol{v} \cdot \boldsymbol{e} \mathrm{d} t$ is a shorthand notation for the distance swan by the swimmer. Notice how in the general case the configuration of the swimmer at time $T$ is a rigid motion of the original configuration $\Gamma(0)$, where $\boldsymbol{R}$ indicates a rotation and $c \boldsymbol{e}$ is the translational vector.

Eq. (1c) states that forces and torques are generated internally by the swimmer. Here $\boldsymbol{\sigma}=\eta\left(\nabla \boldsymbol{u}+\nabla^{T} \boldsymbol{u}\right)-p \boldsymbol{I}$ is the stress in the fluid, with $\boldsymbol{I}$ the identity. 
In the axisymmetric case, the second of Equations $1 \mathrm{cc}$ is automatically satisfied, and we will no longer refer to it. Moreover, due to the symmetry of the system, no rotation is allowed and $\boldsymbol{R}$ is the identity matrix, which we will omit from now on. Notice that this also implies that both velocities and generated force densities are axisymmetric.

Knowledge of the velocity $\boldsymbol{v}(\boldsymbol{x}, t)$ on the boundary is enough to ensure existence and uniqueness of a solution $\boldsymbol{u}(\boldsymbol{x}, t)$ in the entire external domain, provided that $\Gamma(t)$ is maintained regular enough throughout time: a bound-

ary $\Gamma(t)$ of class $\mathcal{C}^{1}$ and boundary data $\boldsymbol{v} \in H^{\frac{1}{2}}(\Gamma(t))$ guarantee existence and uniqueness of the exterior solution $\boldsymbol{u}$ at time $t$, see, for example Ref. [7].

\subsection{Global Hydrodynamic Interactions}

In Equations (1a) we do not describe what happens inside the domain $\Omega(t)$. While in reality micro swimmers have a rather complex internal structure, we will make two simplifying assumptions:

- all movement capabilities of the organism are achieved through changes on the surface $\Gamma(t)$ only;

- the part of the swimmer enclosed by $\Gamma(t)$ contains fluid identical to the one in which the swimmer is immersed, hence the same equations hold in the interior and in the exterior of $\Omega(t)$.

Under these assumptions, a swimmer is fully defined if we either assign a compatible movement of its boundary or by specifying the distribution of stress jumps that the swimmer is sustaining on $\Gamma(t)$.

The underlying principle we are exploiting here is that movement is achieved via hydrodynamic interaction between the surface of the swimmer and the surrounding fluid. Due to the complexity and diversity of the internal structure of biological swimmers, we don't attempt to model it explicitly. We do allow, however, for the presence of a fluid inside $\Omega(t)$ because this is the typical case for bio-swimmers. We assume for simplicity that the viscosities of inner and outer fluids are the same because this allows one to solve the global Stokes problem using only the single layer potential. More precisely, the following relationship holds (see, for example, Ref. [8]):

$$
8 \pi \eta \boldsymbol{u}(\boldsymbol{x})=-\int_{\Gamma} \tilde{\mathcal{S}}(\boldsymbol{x}-\boldsymbol{y}) \llbracket \boldsymbol{\sigma}(\boldsymbol{y}) \boldsymbol{n}(\boldsymbol{y}) \rrbracket \mathrm{d} \Gamma(\boldsymbol{y}), \quad \boldsymbol{x} \in \Re^{3}
$$


where $\tilde{\mathcal{S}}$ is the three-dimensional free-space Stokeslet

$$
\tilde{\mathcal{S}}(\boldsymbol{r}):=\frac{\mathbb{I}}{|\boldsymbol{r}|}+\frac{\boldsymbol{r} \otimes \boldsymbol{r}}{|\boldsymbol{r}|^{3}}
$$

and the fluid flow is solved for in the entire space $\Re^{3}$.

To simplify the notation a little, we introduce the definition of a Neumann to Dirichlet $N D_{\Gamma}$ and its inverse Dirichlet to Neumann map $D N_{\Gamma}$ as the operators that, for each stress jump distribution $\boldsymbol{f}=\llbracket \boldsymbol{\sigma} \boldsymbol{n} \rrbracket$ on $\Gamma(t)$, return the velocity distribution $\boldsymbol{v}$ generated on $\Gamma$ that satisfy Eq. (2), and vice versa, i.e.:

$$
\begin{aligned}
N D_{\Gamma} \boldsymbol{f}=\boldsymbol{v} \Longleftrightarrow D N_{\Gamma} \boldsymbol{v}= & \boldsymbol{f} \Longleftrightarrow \\
& 8 \pi \eta \boldsymbol{v}(\boldsymbol{x})=-\int_{\Gamma} \tilde{\mathcal{S}}(\boldsymbol{x}-\boldsymbol{y}) \boldsymbol{f}(\boldsymbol{y}) \mathrm{d} \Gamma(\boldsymbol{y}), \boldsymbol{x} \in \Gamma .
\end{aligned}
$$

In the examples we consider, we always work with $\mathcal{C}^{1}$ boundaries $\Gamma$. Therefore Eq. (4) makes sense for $\boldsymbol{f} \in H^{-\frac{1}{2}}(\Gamma)$ and gives $\boldsymbol{v} \in H^{\frac{1}{2}}(\Gamma)$.

We remark here that both the Dirichlet to Neumann map and the Neumann to Dirichlet map (4) are translation invariant with respect to the configuration $\Gamma(t)$, i.e.,

$$
\Gamma_{1}=\Gamma_{0}+\boldsymbol{d} \Longrightarrow N D_{\Gamma_{0}} \boldsymbol{f}=N D_{\Gamma_{1}} \boldsymbol{f}, \quad D N_{\Gamma_{0}} \boldsymbol{v}=D N_{\Gamma_{1}} \boldsymbol{v} \quad \forall \boldsymbol{v}, \quad \forall \boldsymbol{f},
$$

for any constant translation vector $\boldsymbol{d}$, i.e., the same stress jump applied to a translation of $\Gamma$ produces identical velocity distributions on $\Gamma$ itself.

Given the above hypothesis, a biological swimmer can then be modeled entirely as a closed surface that is capable of transmitting forces to the external (as well as to the internal) fluid in the form of stress jumps. The time evolution of the swimmer itself is then given by transporting the boundary with the flow field $\boldsymbol{u}(\boldsymbol{x}, t)$ as in Eq. (1b), and the system of equations satisfied 
by the unknown axisymmetric $\boldsymbol{v}$ and $\boldsymbol{f}$ becomes simply

$$
\begin{array}{ll}
D N_{\Gamma(t)} \boldsymbol{v}=\boldsymbol{f} & \forall \boldsymbol{X} \in \Gamma(t) \\
\frac{\partial \boldsymbol{X}}{\partial t}=\boldsymbol{v}(\boldsymbol{X}, t) & \\
\Gamma(T)=\Gamma(0)+\int_{0}^{T} \int_{\Gamma} \boldsymbol{v} \mathrm{d} \Gamma \mathrm{d} t=\Gamma(0)+c \boldsymbol{e} & \\
\int_{\Gamma(t)} \boldsymbol{f}=0, &
\end{array}
$$

for $t \in[0, T]$, where Eq. (6a) effectively replaces the set of Equations (1a) and we already made the simplifications derived from the symmetry of the system: no external torque can be generated by axisymmetric force distributions, and the only possible motion is a translation along the axis of symmetry.

More compactly, system (6) can be written symbolically as

$$
\begin{aligned}
& \int_{\Gamma} D N_{\Gamma}\left(\frac{\partial \Gamma}{\partial t}\right) \cdot \boldsymbol{e} \mathrm{d} \Gamma=0 \quad \forall t \in[0, T] \\
& \Gamma(T)=\Gamma(0)+c \boldsymbol{e} .
\end{aligned}
$$

Using cylindrical coordinates, the configuration of the swimmer, its force density distribution and its velocity field on $\Gamma(t)$ can all be expressed as a rotation around the axis of symmetry (i.e., the $x$ axis) of one-parameter (vector valued) functions (see Figure 1 for an example configuration).

In particular we fix the domain $\mathcal{D}$ of the parameter $s$ to be the interval $[0,1]$. The three-dimensional configuration $\Gamma$ is the image of the function $\tilde{\boldsymbol{X}}$, given by

$$
\tilde{\boldsymbol{X}}(s, \theta)=\left(\begin{array}{c}
x(s) \\
\sigma(s) \cos (\theta) \\
\sigma(s) \sin (\theta)
\end{array}\right)=\boldsymbol{R}(\theta) \boldsymbol{X}(s), \quad \theta \in[0,2 \pi], \quad s \in[0,1],
$$

while the three-dimensional force density and the velocity fields are given by

$$
\tilde{\boldsymbol{f}}(s, \theta)=\left(\begin{array}{c}
f_{x}(s) \\
f_{\sigma}(s) \cos (\theta) \\
f_{\sigma}(s) \sin (\theta)
\end{array}\right)=\boldsymbol{R}(\theta) \boldsymbol{f}(s), \quad \theta \in[0,2 \pi], \quad s \in[0,1]
$$






Figure 1: An example of the instantaneous configuration of an axisymmetric swimmer on the $(x, \sigma)$-plane. The three-dimensional configuration is obtained by a rotation around the $x$-axis of the function $\boldsymbol{X}(s)$.

and

$$
\tilde{\boldsymbol{u}}(s, \theta)=\left(\begin{array}{c}
u_{x}(s) \\
u_{\sigma}(s) \cos (\theta) \\
u_{\sigma}(s) \sin (\theta)
\end{array}\right)=\boldsymbol{R}(\theta) \boldsymbol{u}(s), \quad \theta \in[0,2 \pi], \quad s \in[0,1],
$$

where $\boldsymbol{R}(\theta)$ is defined as

$$
\boldsymbol{R}(\theta)=\left(\begin{array}{cc}
1 & 0 \\
0 & \cos (\theta) \\
0 & \sin (\theta)
\end{array}\right)
$$

To avoid confusion, we will distinguish between three-dimensional functions $\boldsymbol{u}(\boldsymbol{x})$ and their axisymmetric counterparts $\boldsymbol{u}(s)$, identified with their section on the $(x, \sigma)$-plane, by either using symbols with a tilde on top (e.g., $\tilde{\mathcal{S}}$ for the three-dimensional Stokeslet and $\mathcal{S}$ for the axisymmetric one) or by explicitly specifying the domain variable ( $\boldsymbol{x}$ for three-dimensional functions or $s$ for their $(x, \sigma)$ section). 
Our approach is to assign $\Gamma$ through a set of time dependent scalars $\xi(t) \in V \subseteq \Re^{N}$, so that for each time $t$, the curve $\boldsymbol{X}(\xi(t))(s)$ generates an admissible $\Gamma$. We focus on cases where the boundary $\Gamma$ depends smoothly (e.g., analytically) on the parameters $\xi$, and remains of class $\mathcal{C}^{1}$ for all time, $\boldsymbol{X}(s, t)$ is a non self-intersecting, $\mathcal{C}^{1}$ curve on the $(x, \sigma)$-upper half plane $(\sigma \geq$ $0)$, and the surface obtained by its rotation around the $x$ axis is the boundary $\Gamma(t)$ of a domain $\Omega(t)$ such that its complement $\Re^{3} \backslash \Omega(t)$ is connected.

The admissible shapes we treat in this paper are collections of simple closed curves in the $(x, \sigma)$-upper half plane, or open curves in the same half plane which start and end vertically on the $x$ axis.

\subsection{Optimal Swimming as a Constrained Minimization Problem}

While system (6) describes the relationship between the forces $f$ that the swimmer applies to the surrounding flow and its consequent evolution $\boldsymbol{v}$, it gives no information about the optimality of a given swimming stroke.

A natural optimality criterion consists on minimizing the energy dissipated while swimming at a given average velocity $c / T$. In this sense the optimal stroke is the one that minimizes

$$
\mathcal{E}(\boldsymbol{v}):=\int_{0}^{T} \int_{\Gamma(t)} \boldsymbol{v} \cdot D N_{\Gamma} \boldsymbol{v} \mathrm{d} \Gamma \mathrm{d} t,
$$

where $\boldsymbol{v}$ satisfies the set of Equations (6), subject to the constraint

$$
\mathcal{F}_{c}(\boldsymbol{v}):=\int_{0}^{T} \int_{\Gamma(t)} \boldsymbol{v} \cdot \boldsymbol{e} \mathrm{d} \Gamma \mathrm{d} t-c=0 .
$$

In order to embed in the minimization problem also Eq. (6d), it is convenient to express the entire problem in terms of shape changes rather than absolute velocities.

We assume that the changes in the configuration $\Gamma(t)$ of the swimmer happen only through a set of $N+1$ scalar functions of time which we identify as the shape variables $\xi_{i}(t), i=1 \ldots N$, and the location $\varphi(t)$ on the axis of symmetry of a distinguished point.

Moreover, the configuration $\Gamma(\xi, \varphi)$ is known for any admissible shape $\xi$ and displacement $\varphi \in \Re$ and has the form

$$
\Gamma(\xi, \varphi):=\Gamma_{0}(\xi)+\boldsymbol{e} \varphi, \quad(\xi, \varphi) \in \Re^{N} \times \Re,
$$


as exemplified in Figure 2.

In particular, we assume that the configuration $\Gamma$ is the image of a function $\boldsymbol{X}$ of the parameter $s \in \mathcal{D}$, which identifies the location of each material point of $\Gamma$, i.e.,

$$
\boldsymbol{X}(\xi, \varphi, s):=\overline{\boldsymbol{X}}(\xi, s)+\boldsymbol{e} \varphi, \quad \Gamma(\xi, \varphi)=\{\boldsymbol{X}(\xi, \varphi, s), \quad s \in \mathcal{D}\}
$$

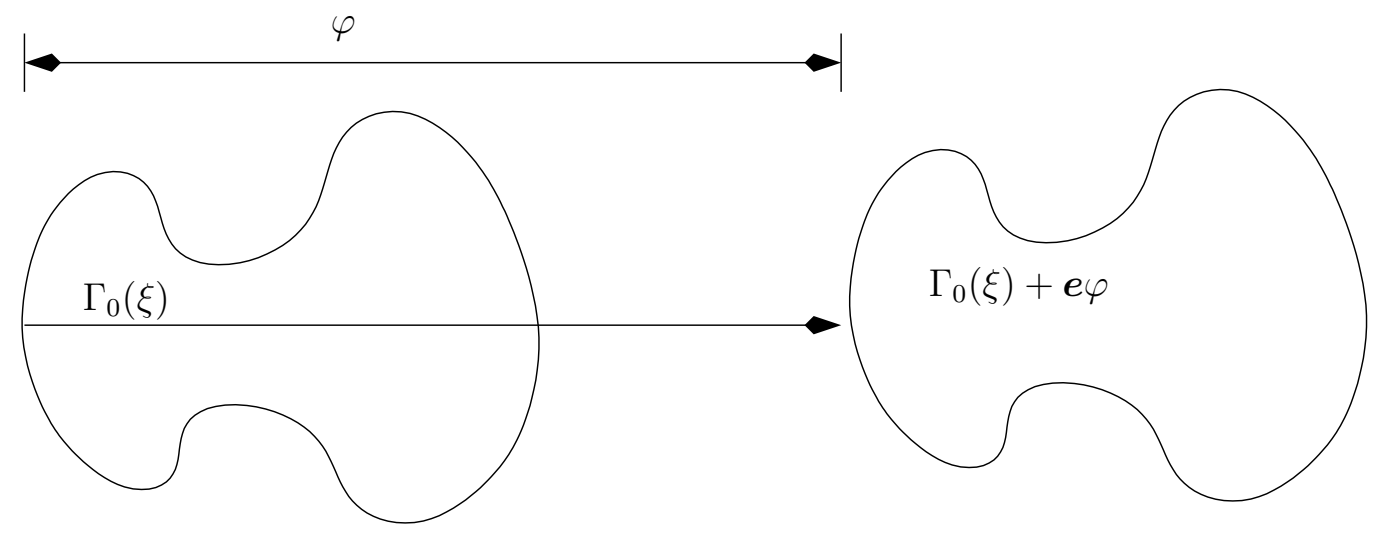

Figure 2: Example of configuration $\Gamma(\xi, \varphi)$.

Using Equations (6b) and (15), the velocity of the swimmer can then be expressed in terms of rate of shape changes $\dot{\xi}$ and in terms of translational velocity $\dot{\varphi}$ as

$$
\begin{aligned}
\boldsymbol{v}(\boldsymbol{X}(\xi(t), \varphi(t), s)) & =\frac{\partial \boldsymbol{X}(\xi, \varphi, s)}{\partial \xi_{i}} \dot{\xi}_{i}(t)+\frac{\partial \boldsymbol{X}(\xi, \varphi, s)}{\partial \varphi} \dot{\varphi}(t) \\
& =\frac{\partial \overline{\boldsymbol{X}}(\xi, s)}{\partial \xi_{i}} \dot{\xi}_{i}(t)+\boldsymbol{e} \dot{\varphi}(t) \\
& =\overline{\boldsymbol{u}}_{i}(s) \dot{\xi}_{i}(t)+\boldsymbol{e} \dot{\varphi}(t), \quad \forall s \text { in } \mathcal{D}, \quad \forall t \in[0, T],
\end{aligned}
$$

where summation is implied on repeated indices. Here $\boldsymbol{e}$ is a unit vector directed along the axis of symmetry, while $\overline{\boldsymbol{u}}_{i}$ is the change in the configuration $\Gamma(t)$ at the position $\boldsymbol{X}(\xi, \varphi, s)$ due to a variation of the shape parameter $\xi_{i}$.

Using this formalism, the periodicity condition (6c) becomes simply

$$
\xi(0)=\xi(T) .
$$


Eq. (6d) implies that

$$
\int_{\Gamma} D N_{\Gamma} \boldsymbol{v} \cdot \boldsymbol{e} \mathrm{d} \Gamma=0
$$

which can be rewritten using Eq. 16 as

$$
\int_{\Gamma} D N_{\Gamma}\left(\overline{\boldsymbol{u}}_{i} \dot{\xi}_{i}+\boldsymbol{e} \dot{\varphi}\right) \cdot \boldsymbol{e}=\mathbb{N}(\xi) \cdot \dot{\xi}+\mathbb{M}(\xi) \dot{\varphi}=0,
$$

where

$$
\begin{aligned}
& \mathbb{N}_{i}(\xi):=\int_{\Gamma} D N_{\Gamma} \overline{\boldsymbol{u}}_{i} \cdot \boldsymbol{e} \mathrm{d} \Gamma \\
& \mathbb{M}(\xi):=\int_{\Gamma} D N_{\Gamma} \boldsymbol{e} \cdot \boldsymbol{e} \mathrm{d} \Gamma .
\end{aligned}
$$

It is now possible to write $\dot{\varphi}$ as a function of $\dot{\xi}$

$$
\dot{\varphi}=\mathbb{V}(\xi) \cdot \dot{\xi}=-\frac{\mathbb{N}(\xi)}{\mathbb{M}(\xi)} \cdot \dot{\xi},
$$

which allows us to express the energy dissipated by a given stroke $\xi$ as

$$
\mathcal{E}(\xi):=\int_{0}^{T} \mathbb{G}(\xi) \dot{\xi} \cdot \dot{\xi} \mathrm{d} t,
$$

where

$$
\mathbb{G}_{i j}(\xi):=\int_{\Gamma} D N_{\Gamma} \overline{\boldsymbol{w}}_{i} \cdot \overline{\boldsymbol{w}}_{j} \mathrm{~d} \Gamma, \quad \overline{\boldsymbol{w}}_{i}:=\overline{\boldsymbol{u}}_{i}+\mathbb{V}_{i}(\xi) \boldsymbol{e} .
$$

A natural space where we can look for solutions $\xi$ of our problem is then given by the Sobolev space

$$
\mathcal{V}:=\left\{\xi \in H^{1}(0, T)^{N}, \text { s.t. } \xi(0)=\xi(T)\right\}
$$

of periodic $H^{1}$ functions of $N$ components. Optimal swimming is then given by the solution $\bar{\xi} \in \mathcal{V}$ of

$$
\min _{\xi \in \mathcal{V}} \int_{0}^{T} \mathbb{G}(\xi) \dot{\xi} \cdot \dot{\xi} \mathrm{d} t=: \mathcal{E}(\xi)
$$

$$
\text { subject to } \quad \int_{0}^{T} \mathbb{V}(\xi) \cdot \dot{\xi} \mathrm{d} t-c=: \mathcal{F}_{c}(\xi)=0 \text {. }
$$


In principle, additional constraints could be added to Eq. 24b to fix, for example, that the swimmer initial and final shape are given, or that the shape never reaches out of given physical bounds (due, for example, to construction constraints in artificial swimmers or physiological constraints in biological swimmers):

$$
\begin{aligned}
\xi(0) & =\xi(T)=\xi_{0} \\
\xi_{L} & \leq \xi(t) \leq \xi_{U}
\end{aligned} \quad \forall t \in[0, T] .
$$

In Ref. [5] and Ref. [6], problem (24) with the addition of constraint 25a has been considered for some special model swimmers. In this case, the space of admissible shapes is

$$
\mathcal{V}_{0}:=\left\{\xi \in H^{1}(0, T)^{N} \text {, s.t. } \xi(0)=\xi(T)=\xi_{0}\right\}
$$

and the following hypothesis guarantees the existence of a solution to problem (24):

There exists $\xi \in \mathcal{V}_{0}$ which satisfies $(24 b)$ and such that $\mathcal{E}(\xi)$ is finite, (27) as shown below.

Proposition 1 Assume $\mathbb{G}$ and $\mathbb{V}$ are continuous functions and that there exist positive constants $\alpha, \beta$ such that

$$
\exists \alpha>0, \beta>0, \text { such that }, \beta|\eta|^{2} \geq \eta \cdot \mathbb{G}(\xi) \eta \geq \alpha|\eta|^{2} \quad \forall \eta \in \Re^{N},
$$

uniformly for admissible shapes $\xi \in \mathcal{V}_{0}$. Then there exists a solution to problem (24).

Proof 1 The proof is a straightforward application of the Direct Method of the Calculus of Variations. Indeed, under the given hypotheses, the functional to minimize is coercive and lower semicontinuous with respect to weak convergence in $\left(H^{1}(0, T)\right)^{N}$. Moreover the constraint is continuous with respect to this topology. Condition (27) ensures that the set over which the minimization is done is not empty.

Remark 1 Condition (27) is nontrivial. It requires that the swimmer can indeed cover the prescribed distance, using finite energy. As explained in Ref. [5] and Ref. [6], this is a controllability condition equivalent to asking that the constraint (24b) is non-holonomic. 
Remark 2 The uniform coercivity condition (28) is a very reasonable condition although very difficult to prove in a framework as general as the one presented here. What is however clear in view of its definition, is that for all $\xi \in \mathcal{V}_{0}$ the matrix $\mathbb{G}(\xi)$ is positive definite.

We will not pursue further the analysis of problem (24), which requires analytical verification of conditions such as (27) and (28) on a case-by-case basis. In the rest of our work, we will focus instead on strategies to find solutions to the optimization problem (24) numerically.

\section{Numerical Approximation}

The two main ingredients of the computer simulation of the swimmer problem are an efficient numerical implementation of the Dirichlet to Neumann map (4) and a strategy for the search of minima of $\mathcal{E}(\xi)$ in Eq. (24) satisfying the constraints.

We solve the first problem using the boundary element method (with a custom code written in $\mathrm{C}++$, using the deal. II library [9, 10] and based on the formulation presented in Ref. [8]). We address the solution to the constrained minimization problem using the reduced space successive quadratic programming strategy (rSQP)[11] provided with the Moocho package of the Trilinos C++ library. [12]

\subsection{Axisymmetric Boundary Integral Equations for Stokes Flow}

Our boundary integral formulation for axisymmetric Stokes flow follows closely Ref. [8]: if we consider Stokes equation in free-space associated with a forcing term due to a Dirac mass centered in $\boldsymbol{y}$ and weighted with the force vector $\boldsymbol{b}$, i.e.,

$$
\begin{array}{ll}
-\eta \Delta \boldsymbol{u}(\boldsymbol{x})+\nabla p(\boldsymbol{x})=-\nabla \cdot \boldsymbol{\sigma}=\boldsymbol{b} \boldsymbol{\delta}(\boldsymbol{x}-\boldsymbol{y}) & \text { in } \Re^{3} \\
\nabla \cdot \boldsymbol{u}(\boldsymbol{x})=0 & \text { in } \Re^{3} \\
\lim _{|\boldsymbol{x}| \rightarrow \infty}|\boldsymbol{u}(\boldsymbol{x})|=0 & \\
\lim _{|\boldsymbol{x}| \rightarrow \infty}|p(\boldsymbol{x})|=0, &
\end{array}
$$


we can express the solutions $\boldsymbol{u}, p$ and $\boldsymbol{\sigma}$ using the free-space Green's functions $\tilde{\mathcal{S}}, \tilde{\boldsymbol{P}}$ and $\tilde{\boldsymbol{T}}$ :

$$
\begin{aligned}
\boldsymbol{u}(\boldsymbol{x}) & =\frac{1}{8 \pi \eta} \tilde{\mathcal{S}}(\boldsymbol{x}-\boldsymbol{y}) \boldsymbol{b} \\
p(\boldsymbol{x}) & =\frac{1}{8 \pi} \tilde{\boldsymbol{P}}(\boldsymbol{x}-\boldsymbol{y}) \cdot \boldsymbol{b} \\
\boldsymbol{\sigma}(\boldsymbol{x}) & =\frac{1}{8 \pi} \tilde{\boldsymbol{T}}(\boldsymbol{x}-\boldsymbol{y}) \boldsymbol{b}
\end{aligned}
$$

where

$$
\begin{aligned}
\tilde{\mathcal{S}}_{i j}(\boldsymbol{r}) & =\frac{\delta_{i j}}{|\boldsymbol{r}|}+\frac{r_{i} r_{j}}{|\boldsymbol{r}|^{3}} \\
\tilde{P}_{i}(\boldsymbol{r}) & =2 \frac{r_{i}}{|\boldsymbol{r}|^{3}} \\
\tilde{T}_{i j k}(\boldsymbol{r}) & =-6 \frac{r_{i} r_{j} r_{k}}{|\boldsymbol{r}|^{5}} .
\end{aligned}
$$

The axisymmetric approximation allows us to reduce the swimmer problem to a boundary integral equation on a one-dimensional curve, i.e., the swimmer is allowed to generate only axial-symmetric surface forces, which are then transmitted to the fluid.

We introduce the following notation for integrals on the surface $\Gamma$, identified by the configuration $\boldsymbol{X}(s)=(X(s), \sigma(s)), s \in[0,1]$ :

$$
\begin{aligned}
\int_{\Gamma} \tilde{g} \mathrm{~d} \Gamma & =2 \pi \int_{0}^{1} g(s) J_{X}(s) \sigma(s) \mathrm{d} s \\
& =: \int_{\boldsymbol{X}(s)} g(s) \mathrm{d} s
\end{aligned}
$$

where we indicated with $J_{X}(s)=\left|\frac{\partial X}{\partial s}(s)\right|$, and the last line serves only to ease the notation.

To solve Stokes equations in the axisymmetric case, we integrate analytically the singular integral Eq. (2) around the axis of rotation, while keeping the point $\boldsymbol{x}$ on the half-plane $\theta=0$.

The azimuthal integration gives rise to a single layer potential kernel, which can be expressed using complete elliptic integrals of the first and second kind and produces a linearly coupled set of one-dimensional integral equations. 
We can write the resulting equation as

$$
8 \pi \eta \boldsymbol{v}_{\alpha}(q)=-\int_{\boldsymbol{X}(s)} \mathcal{S}_{\alpha \beta}(\boldsymbol{X}(q), \boldsymbol{X}(s)) \boldsymbol{f}_{\beta}(s) \mathrm{d} s
$$

where the indices $\alpha$ and $\beta$ are either $x$ or $\sigma$, to indicate the distance along the axis of symmetry, or the distance from the axis itself.

The axisymmetric kernel $\mathcal{S}$ represents the single layer integrals of a ring of singularities passing through the point $\tilde{\boldsymbol{X}}(s, 0)=\boldsymbol{R}(0) \boldsymbol{X}(s)$ :

$$
\mathcal{S}(\boldsymbol{X}, \boldsymbol{Y}):=\int_{0}^{2 \pi}\left[\begin{array}{ll}
\tilde{\mathcal{S}}_{x x} & \left(\tilde{\mathcal{S}}_{y x} \cos \theta+\tilde{\mathcal{S}}_{z x} \sin \theta\right) \\
\tilde{\mathcal{S}}_{x y} & \left(\tilde{\mathcal{S}}_{y y} \cos \theta+\tilde{\mathcal{S}}_{z y} \sin \theta\right)
\end{array}\right] \mathrm{d} \theta
$$

where $\tilde{\mathcal{S}}=\tilde{\mathcal{S}}(\boldsymbol{R}(0) \boldsymbol{X}, \boldsymbol{R}(\theta) \boldsymbol{Y})$ is the three-dimensional Stokeslet given in 31, evaluated at the points $\boldsymbol{R}(0) \boldsymbol{X}$ and $\boldsymbol{R}(\theta) \boldsymbol{Y}$.

The above integration can be expressed in terms of complete elliptic integrals of the first and second kind, which are defined, respectively, as

$$
K(x):=\int_{0}^{\pi / 2} \frac{\mathrm{d} \theta}{\sqrt{1-x^{2} \sin ^{2} \theta}} \mathrm{d} \alpha, \quad x \in[0,1),
$$

which is a logarithmically singular integral, and

$$
E(x):=\int_{0}^{\pi / 2} \sqrt{1-x^{2} \sin ^{2} \theta} \mathrm{d} \theta, \quad x \in[0,1],
$$

which is a bounded integral.

Indeed, if we define

$$
\boldsymbol{X}(q)=\left(\begin{array}{c}
x_{0} \\
\sigma_{0}
\end{array}\right), \quad r:=\sqrt{\left(x-x_{0}\right)^{2}+\left(\sigma-\sigma_{0}\right)^{2}}, \quad \Delta x=x-x_{0},
$$

and

$$
k^{2}=\frac{4 \sigma \sigma_{0}}{\left(x-x_{0}\right)^{2}+\left(\sigma+\sigma_{0}\right)^{2}}
$$


then the axisymmetric Stokeslet is given by (see Ref. [8])

$$
\begin{aligned}
& \mathcal{S}_{x x}=2 k \sqrt{\frac{\sigma}{\sigma_{0}}}\left(K(k)+\Delta x^{2} \frac{E(k)}{r^{2}}\right) \\
& \mathcal{S}_{x \sigma}=+\frac{k \Delta x}{\sigma} \sqrt{\frac{\sigma}{\sigma_{0}}}\left(K(k)-\left(\sigma_{0}^{2}-\sigma^{2}+\Delta x^{2}\right) \frac{E(k)}{r^{2}}\right) \\
& \mathcal{S}_{\sigma x}=-\frac{k \Delta x}{\sigma_{0}} \sqrt{\frac{\sigma}{\sigma_{0}}}\left(K(k)+\left(\sigma_{0}^{2}-\sigma^{2}-\Delta x^{2}\right) \frac{E(k)}{r^{2}}\right) \\
& \mathcal{S}_{\sigma \sigma}=\frac{k}{\sigma_{0} \sigma} \sqrt{\frac{\sigma}{\sigma_{0}}}\left(\left(\sigma_{0}^{2}+\sigma^{2}+2 \Delta x^{2}\right) K(k)-\right. \\
& \left.\left(2 \Delta x^{4}+3 \Delta x^{2}\left(\sigma_{0}^{2}+\sigma^{2}\right)+\left(\sigma_{0}^{2}-\sigma^{2}\right)^{2}\right) \frac{E(k)}{r^{2}}\right),
\end{aligned}
$$

where the diagonal entries are singular and behave like $\mathcal{S}_{x x} \sim \mathcal{S}_{\sigma \sigma} \sim-2 \ln (r)$.

\subsection{Spatial Discretization}

We employ an iso-parametric finite dimensional representation for the spatial discretization of the configuration $\boldsymbol{X}$, and of the velocity and force density $\boldsymbol{u}$ and $\boldsymbol{f}$. We call $V_{h}=\operatorname{span}\left\{\boldsymbol{\tau}^{i}\right\}_{1 \leq i \leq M}$ the finite dimensional space that we use for the discretization. To ensure regularity and smoothness of the boundary $\Gamma$ for any $t$, we choose a discretization based on cubic bell splines.

In particular at any given time we identify elements of $V_{h}$ with the coefficients of $M$ dimensional vectors, as in

$$
\begin{aligned}
\boldsymbol{X}_{h}(s) & =\boldsymbol{X}^{i} \boldsymbol{\tau}^{i}(s) \\
\boldsymbol{v}_{h}(s) & =\boldsymbol{v}^{i} \boldsymbol{\tau}^{i}(s),
\end{aligned}
$$

where we used the summation convention, and $i$ goes from 1 to $M$. The superscripts here indicate the indices of the basis functions that span the space $V_{h}$. When confusion cannot arise, we will use $\boldsymbol{X}_{h}$ without " $(s)$ " to indicate the $\Re^{M}$ vector of components $\boldsymbol{X}^{i}, i=1 \ldots M$ which uniquely identify the vector valued function $\boldsymbol{X}_{h}(s)$ in $V_{h}$ as in (38).

To take into account collections of shapes, it is possible to introduce controlled discontinuities in the basis functions $\boldsymbol{\tau}^{i}(s)$, by overlapping a sufficient number of nodes in the knot-span that generates the B-Spline basis, as explained, for example, in Ref. [13]. 
The Galerkin approach to the boundary element method can be understood by multiplying Eq. (33) on both sides with test functions (or virtual forces) $\boldsymbol{\tau}$ and integrating on $\Gamma$

$$
\begin{aligned}
& \int_{\boldsymbol{X}(s)} \boldsymbol{v}(s) \cdot \boldsymbol{\tau}(s) \mathrm{d} s= \\
&-\int_{\boldsymbol{X}(q)} \boldsymbol{\tau}(q) \cdot \frac{1}{8 \pi \eta} \int_{\boldsymbol{X}(s)} \mathcal{S}(\boldsymbol{X}(q), \boldsymbol{X}(s)) \boldsymbol{f}(s) \mathrm{d} s \mathrm{~d} q \\
& \forall \boldsymbol{\tau} \in C^{\infty}([0,1])^{2}
\end{aligned}
$$

which, on a discrete level, yields the discrete version of the Dirichlet to Neumann map:

$$
\boldsymbol{f}_{h}=\mathcal{A}^{-1} \mathcal{M} \boldsymbol{u}_{h}
$$

where both matrices $\mathcal{A}$ and $\mathcal{M}$ depend on $\boldsymbol{X}_{h}(s)$ and are defined as

$$
\mathcal{A}^{i j}=-\int_{\boldsymbol{X}_{h}(q)} \boldsymbol{\tau}^{i}(q) \cdot \frac{1}{8 \pi \eta} \int_{\boldsymbol{X}_{h}(s)} \mathcal{S}\left(\boldsymbol{X}_{h}(q), \boldsymbol{X}_{h}(s)\right) \boldsymbol{\tau}^{j}(s) \mathrm{d} s \mathrm{~d} q,
$$

and

$$
\mathcal{M}^{i j}=\int_{\boldsymbol{X}_{h}(s)} \boldsymbol{\tau}^{j}(s) \cdot \boldsymbol{\tau}^{i}(s) \mathrm{d} s .
$$

Numerical computation of the matrix entries of both $\mathcal{A}$ and $\mathcal{M}$ is performed using high order Gauss quadrature formulae, as well as Gauss-Log quadrature formulae for the diagonal entries of $\mathcal{A}$, in order to properly take into account the logarithmic singularity of the axisymmetric Stokeslet (see, for example, Ref. [8, 14, 15]).

Notice that, once we have two vectors $\boldsymbol{f}_{h}$ and $\boldsymbol{v}_{h}$ in $\Re^{M}$ that represent objects of $V_{h}$, we can compute their integrals on $\boldsymbol{X}_{h}(s)$ by scalar product in $\Re^{M}$ through the matrix $\mathcal{M}$ :

$$
\int_{\boldsymbol{X}(s)} \boldsymbol{f}_{h}(s) \cdot \boldsymbol{v}_{h}(s) \mathrm{d} s=\boldsymbol{f}_{h} \cdot \boldsymbol{M} \boldsymbol{v}_{h}=\boldsymbol{v}_{h} \cdot \boldsymbol{M} \boldsymbol{f}_{h}=\boldsymbol{v}^{i} \mathcal{M}^{i j} \boldsymbol{f}^{j}
$$

where in the left hand side $(\cdot)$ has the meaning of scalar product between vector functions of $\Re^{2}$, while on the right hand sides $(\cdot)$ should be read as the scalar product in $\Re^{M}$. 
Once we know how to discretize a domain $\Gamma$ and write a discrete version of the Dirichlet to Neumann Map for any configuration, we simply define the discrete shape basis functions as the counter part of $\partial \overline{\boldsymbol{X}}(\xi, \varphi) / \partial \xi$, i.e.,

$$
\begin{aligned}
\overline{\boldsymbol{u}}_{h}^{i}(s) & =\frac{\partial \overline{\boldsymbol{X}}_{h}(\xi, s)}{\partial \xi_{i}} \quad i=1 \ldots N \\
\boldsymbol{e}_{h}(s) & =\boldsymbol{e}(s)
\end{aligned}
$$

where $\overline{\boldsymbol{X}}(\xi, s)$ indicates a point on the $(x, \sigma)$-half-plane of the configuration $\Gamma_{0}(\xi)$.

The discrete shape basis functions $\overline{\boldsymbol{u}}_{h}^{i}(s)$ are simply constructed as the b-spline interpolation of their continuous counter parts, which are a datum of the problem, while the discrete basis function $\boldsymbol{e}_{h}(s)$ for the displacement vector space is simply equal to the discrete representation of the constant unit vector directed along the axis of symmetry.

Given $N+1$ arbitrary time functions $(\xi(t), \varphi(t))$, it is then possible to build the discrete configuration $\boldsymbol{X}_{h}(\xi(t), \varphi(t))$, the discrete basis functions for the rate of shape change $\overline{\boldsymbol{u}}_{h}^{i}$ and to compute the Dirichlet to Neumann map for arbitrary data defined on the discrete curve.

In particular we can compute the discrete versions of $\mathbb{V}$ and $\mathbb{G}$ as

$$
\mathbb{V}_{i, h}(\xi):=-\frac{\left(\mathcal{A}^{-1} \mathcal{M} \bar{u}_{h}^{i}(\xi)\right) \cdot \mathcal{M} \boldsymbol{e}_{h}}{\left(\mathcal{A}^{-1} \mathcal{M} \boldsymbol{e}_{h}\right) \cdot \mathcal{M} \boldsymbol{e}_{h}}
$$

and

$$
\mathbb{G}_{i j, h}(\xi):=\left(\mathcal{A}^{-1} \mathcal{M}\left(\overline{\boldsymbol{u}}_{h}^{i}(\xi)+\mathbb{V}_{i, h}(\xi) \boldsymbol{e}_{h}\right)\right) \cdot \boldsymbol{\mathcal { M }}\left(\overline{\boldsymbol{u}}_{h}^{j}(\xi)+\mathbb{V}_{j, h}(\xi) \boldsymbol{e}_{h}\right)
$$

The semi-discrete formulation of the optimal swimming problem is simply obtained by substituting in Eq. (24) $\mathbb{V}$ and $\mathbb{G}$ with their spatial discrete counterparts:

$$
\begin{array}{cl}
\min _{\xi \in \mathcal{V}} & \int_{0}^{T} \mathbb{G}_{h}(\xi) \dot{\xi} \cdot \dot{\xi} \mathrm{d} t=: \mathcal{E}_{h}(\xi) \\
\text { subject to } & \int_{0}^{T} \mathbb{V}_{h}(\xi) \cdot \dot{\xi} \mathrm{d} t-c=: \mathcal{F}_{h}^{c}(\xi)=0 .
\end{array}
$$




\subsection{Time Discretization}

The dependency of the domain $\Gamma$ (or, equivalently, of its axisymmetric representation $\boldsymbol{X}$ ) on the time variable $t$ is only through the shape and position functions $\xi_{i}(t)$ and $\varphi(t)$. In particular we rewrote the problem in a way that makes it only dependent on the shape parameters $\xi \in \mathcal{V}$.

We approximate also these functions with cubic b-spline functions, with a possibly different dimension $Q$. In particular we have

$$
\mathcal{V}_{h}=\left\{\xi_{h}(t) \in\left(\operatorname{span}\left\{\gamma^{\alpha}(t)\right\}_{\alpha=1}^{Q}\right)^{N}, \text { s.t. } \xi_{h}(0)=\xi_{h}(T)\right\},
$$

where we use Greek letters to distinguish basis functions referring to the time

variable $t$ from those referring to the space variable $s$ and again we can write

$$
\xi_{h}(t)_{i}=\xi_{i}^{\alpha} \gamma^{\alpha}(t) \quad i=1 \ldots N
$$

where summation is implied on the repeated indices $\alpha$ which go from 1 to $Q$.

Notice that a numerical approximation $\xi_{h}$ is now a vector of $\Re^{N \times Q}$, which we identify either with two indices $\xi_{i}^{\alpha}$, or with a single capital letter index $I=1 \ldots N \times Q$, where

$$
I:=i+(\alpha-1) N, \quad \xi_{h}:=\left\{\left\{\xi_{i}^{\alpha}\right\}_{i=1}^{N}\right\}_{\alpha=1}^{Q}=\left\{\xi_{I}\right\}_{I=1}^{N \times Q},
$$

that is

$$
\xi_{h}:=\left[\xi_{1}^{1}, \xi_{2}^{1}, \ldots, \xi_{N}^{1}, \quad \xi_{1}^{2}, \xi_{2}^{2}, \ldots, \xi_{N}^{2}, \quad \ldots, \quad \xi_{1}^{Q}, \xi_{2}^{Q}, \ldots, \xi_{N}^{Q}\right]^{\prime}
$$

\subsection{Discrete Optimal Swimming}

With the discretization $\mathcal{V}_{h}$ of the infinite dimensional space $\mathcal{V}$, we can reduce the minimization problem to a finite dimensional one defined on $\Re^{Q \times N}$, where $Q$ is the total number of basis splines selected for the discretization of the $N$ shape parameters in time, and the fully discrete problem becomes

$$
\begin{aligned}
\min _{\xi_{h} \in \mathcal{V}_{h}} & \mathcal{E}_{h}\left(\xi_{h}\right):=\mathfrak{E}\left(\xi_{h}\right) \xi_{h} \cdot \xi_{h} \\
\text { subject to } & \mathcal{F}_{h}^{c}\left(\xi_{h}\right):=\mathfrak{F}\left(\xi_{h}\right) \cdot \xi_{h}-c=0,
\end{aligned}
$$


where the matrix $\mathfrak{E}\left(\xi_{h}\right)$ in $\Re^{(N \times Q) \times(N \times Q)}$ and the vector $\mathfrak{F}\left(\xi_{h}\right)$ in $\Re^{(N \times Q)}$ are defined as

$$
\begin{aligned}
\mathfrak{E}_{I J}\left(\xi_{h}\right) & :=\int_{0}^{T} \mathbb{G}_{i j}\left(\xi_{h}(t)\right) \dot{\gamma}^{\alpha}(t) \cdot \dot{\gamma}^{\beta}(t) \mathrm{d} t \\
\mathfrak{F}_{I}\left(\xi_{h}\right) & :=\int_{0}^{T} \mathbb{V}_{i}\left(\xi_{h}(t)\right) \dot{\gamma}^{\beta}(t) \mathrm{d} t,
\end{aligned}
$$

and the relationship between the indices $I, J$ and $i, j, \alpha, \beta$ is given by (50) and $J=j+(\beta-1) N$.

The problem is now written in the framework of classical finite dimensional optimization. Indeed, if we define the nabla operator $\nabla$ applied to a function of the vector variable $\xi_{h}$ as

$$
\left(\nabla \boldsymbol{F}\left(\xi_{h}\right)\right)_{I}:=\frac{\partial \boldsymbol{F}\left(\xi_{h}\right)}{\partial \xi_{I}},
$$

we can then write the Lagrangian $\mathcal{L}\left(\xi_{h}, \lambda\right)$ and its derivatives associated with problem (52) as

$$
\begin{aligned}
\mathcal{L}\left(\xi_{h}, \lambda\right) & :=\mathcal{E}_{h}\left(\xi_{h}\right)+\lambda \mathcal{F}_{h}^{c}\left(\xi_{h}\right) \\
\nabla \mathcal{L}\left(\xi_{h}, \lambda\right) & :=\nabla \mathcal{E}_{h}\left(\xi_{h}\right)+\lambda \nabla \mathcal{F}_{h}^{c}\left(\xi_{h}\right) \\
\nabla^{2} \mathcal{L}\left(\xi_{h}, \lambda\right) & :=\nabla^{2} \mathcal{E}_{h}\left(\xi_{h}\right)+\lambda \nabla^{2} \mathcal{F}_{h}^{c}\left(\xi_{h}\right) .
\end{aligned}
$$

The first and second-order necessary Karush-Kuhn-Tucker (KKT) optimality conditions for a solution $\left(\bar{\xi}_{h}, \bar{\lambda}\right)$ to $(52)$ are:

$$
\begin{aligned}
& \nabla \mathcal{L}\left(\bar{\xi}_{h}, \bar{\lambda}\right)=\nabla \mathcal{E}_{h}\left(\bar{\xi}_{h}\right)+\bar{\lambda} \nabla \mathcal{F}_{h}^{c}\left(\bar{\xi}_{h}\right)=0 \\
& \mathcal{F}_{h}^{c}\left(\bar{\xi}_{h}\right)=0 \\
& \nabla^{2} \mathcal{L}\left(\bar{\xi}_{h}, \bar{\lambda}\right) \eta \cdot \eta \quad \geq 0 \quad \forall \eta \in \mathcal{V}_{h},
\end{aligned}
$$

also known as linear dependence of gradients, feasibility and curvature conditions.

A popular class of methods for solving non linear constrained minimization problems is successive quadratic programming (SQP) (see, e.g., Ref. [11]), which is equivalent to applying Newton's method to solve the the minimality conditions (56).

At each Newton iteration $k$ for (56), the linear subproblem (also known as the KKT system) takes the form

$$
\left(\begin{array}{cc}
W & A \\
A^{T} & 0
\end{array}\right)\left(\begin{array}{c}
d \\
d_{\lambda}
\end{array}\right)=-\left(\begin{array}{c}
\nabla \mathcal{L}^{(k)} \\
\mathcal{F}_{c}^{(k)}
\end{array}\right),
$$


where

$$
\begin{aligned}
d & :=\xi_{h}^{(k+1)}-\xi_{h}^{(k)} \\
d_{\lambda} & :=\lambda^{(k+1)}-\lambda^{(k)} \\
W & :=\nabla^{2} \mathcal{L}\left(\xi_{h}^{(k)}, \lambda^{(k)}\right) \\
A & :=\nabla \mathcal{F}_{h}^{c}\left(\xi_{h}^{(k)}\right) \\
\mathcal{L}^{(k)} & :=\mathcal{L}\left(\xi_{h}^{(k)}, \lambda^{(k)}\right) \\
\mathcal{F}_{c}^{(k)} & :=\mathcal{F}_{h}^{c}\left(\xi_{h}^{(k)}\right),
\end{aligned}
$$

and we use superscripts between parenthesis $(k)$ to refer to the $k$-th Newton iteration, not to be confused with the index of the b-spline basis functions.

Once we obtained a new estimate of the solution $\left(\xi_{h}^{(k+1)}, \lambda^{(k+1)}\right)$, the error in the optimality conditions (56) is checked. If these KKT errors are within some specified tolerance, the algorithm is terminated with the optimal solution.

If the KKT error is too large, the functions and gradients are then computed at the new point $\xi_{h}^{(k+1)}$ and another KKT subproblem (57) is solved which generates another increment $d$, until convergence or failure.

A successful application of the SQP method requires one to provide exact information about the hessian of the Lagrangian $W$. While this ensures second order convergence of the SQP method, it is not feasible in many practical cases, including ours, where the direct computation of $W$ is overly expensive.

To address this issue, we use a reduced-space SQP (rSQP) method. In rSQP methods, the full-space QP subproblem (57) is decomposed into two smaller subproblems, where the optimization vector variable $\xi_{h}$ is split into dependent and independent variables, by using the linearized equality constraint as a mean to express the dependent variables (often referred to as the control variables) in terms of the independent ones (also referred to as the state variables).

Using this decomposition, the two subproblems can be solved effectively by using an approximation of $W$. We use the implementation of the rSQP method included in the Moocho package of the Trilinos library. [16] 


\section{Numerical Results}

The goal of this section is to present some results obtained with our code. In section 4.1 we present our results for the optimal stroke of the Golestanian swimmer. These should be confronted with the results already presented in Ref. [5] and Ref. [6] with a completely different approach both for the resolution of Stokes equations and for the minimization of the expended energy, which we take as our reference results.

While from the quantitative point of view we observe some marginal differences, qualitatively the method presented in this paper is capable of reproducing the optimal strokes obtained in the previously mentioned works.

We should mention here that the main differences lay in the fact that in those works the approximation of the $\mathbb{V}(\xi)$ and $\mathbb{G}(\xi)$ fields was performed using a Finite Element Method for the simulation of axisymmetric Stokes Flow in a box that was taken large compared to the dimension of the swimmer, but of finite dimension. In comparison, the method we present here is based on the use of Boundary Integral Approximation, which should yield more accurate results on infinite domains such as the one we are interested on.

As a second remark, we observe that the optimal stroke in Ref. [5] and Ref. [6] is obtained by writing explicitly the Euler-Lagrange equation for the constrained optimization problem, and by solving the resulting system of ODEs with an explicit Runge Kutta method, coupled with a shooting approach in order to enforce the periodicity of the strokes.

In this paper, on the other hand, the solution of the minimization problem itself is left to an external library which explores the space of shapes in a very efficient and flexible way, allowing us to solve problems that could not be addressed before. In particular we can solve problems in which we include inequality constraints on the shape variables $\xi$, and we can release the constraints on the initial and final shape, letting the minimizer find the optimal starting shape for our swimmers.

In the experiments that follow, we use water at room temperature $\left(20^{\circ}\right.$ C) as the surrounding fluid, and we express lengths in millimeters $(\mathrm{mm})$, time in seconds $(s)$ and weight in milligrams $(m g)$. Using these units, the viscosity of water is approximately one $\left(1 \mathrm{mPas}=1 \mathrm{mg} \mathrm{mm}^{-1} \mathrm{~s}^{-1}\right)$, and the energy is expressed in pico-Joules $\left(1 p J=1 \mathrm{mg} \mathrm{mm}^{2} \mathrm{~s}^{-2}=10^{-12} \mathrm{~J}\right)$.

The tests were performed on a Macbook Pro, with $2.16 \mathrm{GHz}$ Intel Core 2 Duo processor and 2 GB $667 \mathrm{MHz}$ DDR2 SDRAM. The average running 


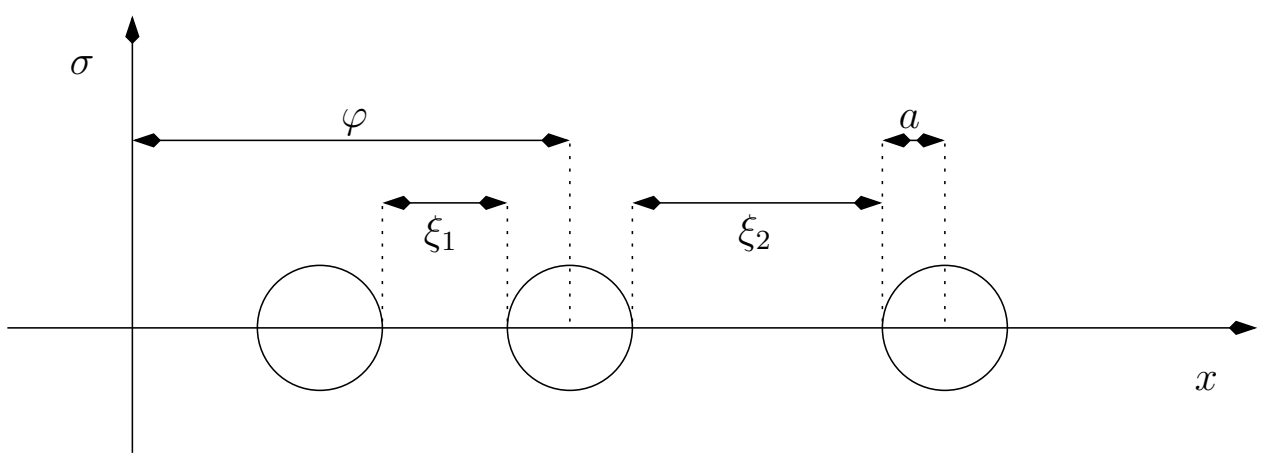

Figure 3: Definition of the Three Sphere Swimmer.

time for the experiments presented in Sec. 4.1 is about 30 seconds, with a maximum memory consumption of $64 \mathrm{MB}$, while the experiments of Sec. 4.2 required on average 10 seconds, with roughly the same memory consumption.

The low running time is possible thanks to the use of cubic B-Spline, which allows one to obtain accurate solutions using very few degrees of freedom. In all the experiments we present, we used 15 control points on each portion of the swimmer for the spatial discretization (i.e., 45 total control points for the three sphere swimmer and 30 for the stick and donut swimmer) and 15 control points for the time discretization of each shape and position variable.

\subsection{Three Sphere Swimmer}

The three sphere swimmer is among the simplest axisymmetric swimmers one can think of. It consists of three linked spheres that can only vary their reciprocal distance. The swimmer is completely defined once we have the radius of the three spheres (which we assume to be the same for simplicity), and the location of the three centers of the spheres on the axis of the movement (one size parameter and three positional variables).

Equivalently, we can use the more convenient representation given by the location of the center of the central sphere $(\varphi)$ and the distances between the two lateral spheres and the central one $\left(\xi_{1}\right.$ and $\left.\xi_{2}\right)$, as shown in Figure 3 . These two different representations are clearly equivalent (see, e.g., Ref. [5]). 
Using this convention $\boldsymbol{X}(\xi, \varphi, s)$ can be parametrized as

$$
\begin{aligned}
& \boldsymbol{X}(\xi, \varphi, s)=\left(\begin{array}{c}
\varphi-\xi_{1}-a(2+\cos (3 \pi s)) \\
a \sin (3 \pi s)
\end{array}\right) \quad \text { if } s \in[0,1 / 3) \\
& \boldsymbol{X}(\xi, \varphi, s)=\left(\begin{array}{c}
\varphi-a \cos (3 \pi(s-1 / 3)) \\
a \sin (3 \pi(s-1 / 3))
\end{array}\right) \\
& \boldsymbol{X}(\xi, \varphi, s)=\left(\begin{array}{c}
\varphi+\xi_{2}+a(2-\cos (3 \pi s)) \\
a \sin (3 \pi(s-2 / 3))
\end{array}\right) \quad \text { if } s \in[1 / 3,2 / 3]
\end{aligned}
$$

which implies that the basis functions for shape changes $\overline{\boldsymbol{u}}_{i}:=\partial \boldsymbol{X} / \partial \xi_{i}$ are defined as

$$
\overline{\boldsymbol{u}}_{1}(s)=-\chi_{[0,1 / 3)}(s) \boldsymbol{e}, \quad \overline{\boldsymbol{u}}_{2}(s)=\chi_{(2 / 3,1]}(s) \boldsymbol{e},
$$

where $\chi_{A}(s)$ is the function which is one if $s \in A$, and zero otherwise. We constrain the shape variables $\xi_{i}$ to be in the interval $[0,6 a]$. Notice that in this paper $\xi$ is the touching distance between the spheres, and not the distance between the centers of the spheres, as in Ref. [5].

In Figure 4 we show the corners of the box $[0,6 a]^{2}$ in which the three sphere swimmer shape is constrained to stay, for a swimmer of radius $a=$ $.05 \mathrm{~mm}$.
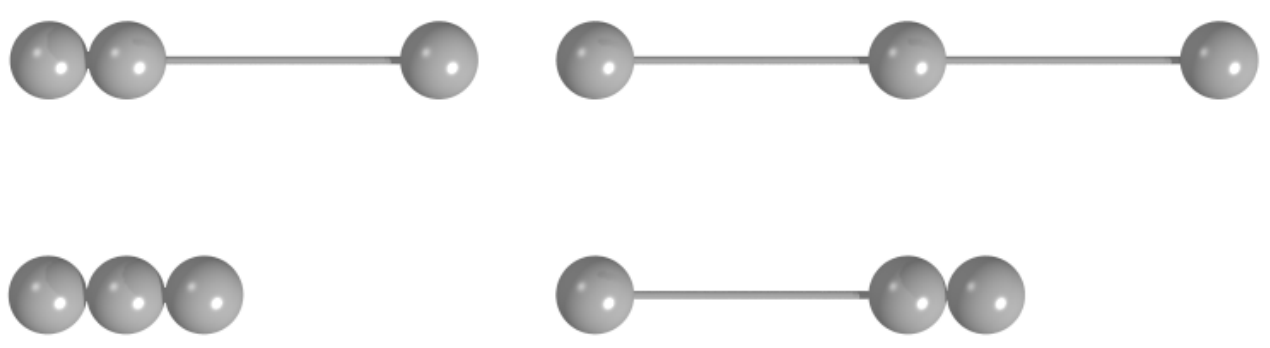

Figure 4: Extremal shape configurations for a Three-Sphere swimmer of radius $a=.05 \mathrm{~mm}$, from left to right and from bottom to top: $\xi=(0,0)$, $\xi=(6 a, 0), \xi=(0,6 a)$ and $\xi=(6 a, 6 a)$.

The basis function for the change in position, $\partial \boldsymbol{X} / \partial \varphi$ is simply equal to the unit vector $\boldsymbol{e}$. In Figure 5, we show the three basis functions that 
allow one to fully describe the system, together with their Dirichlet to Neumann maps, evaluated at the configuration $\xi=(.05 \mathrm{~mm}, .05 \mathrm{~mm})$, which is equivalent to asking that the distance between the centers of the spheres is $3 a$.
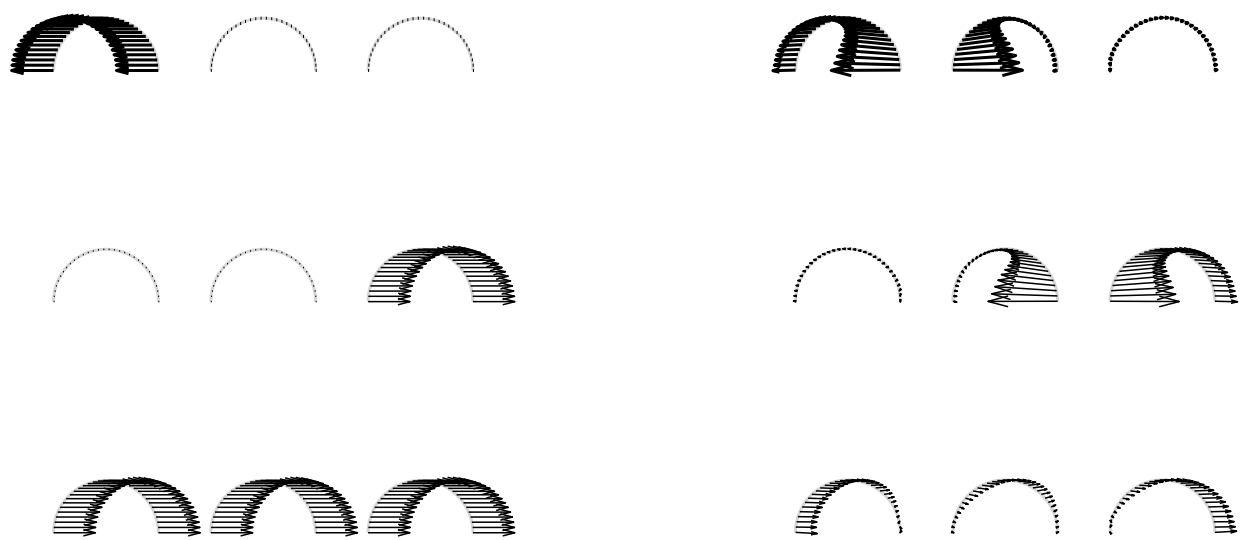

Figure 5: Basis function $\overline{\boldsymbol{u}}_{i}(s)$ and $\boldsymbol{e}$ (left) and their Dirichlet to Neumann maps (right) for $\xi=(a, a)$.
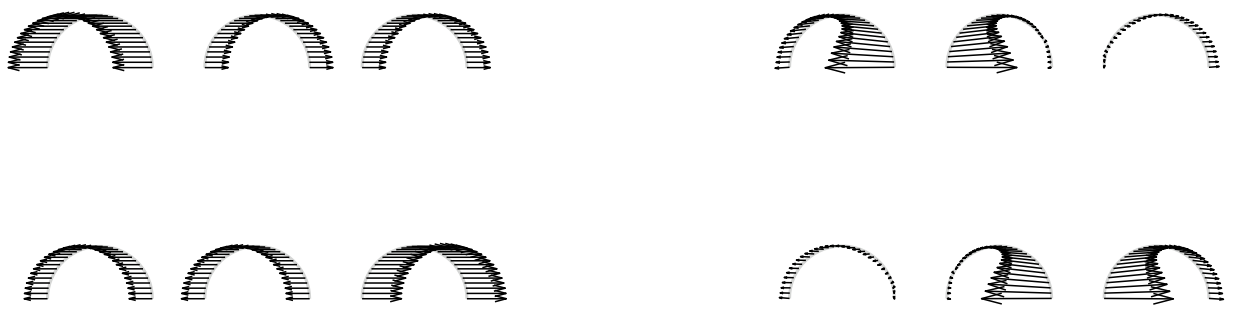

Figure 6: The force-free basis function $\overline{\boldsymbol{w}}_{i}(s)$ (left) and their Dirichlet to Neumann maps (right) for $\xi=(a, a)$.

Once we have the various basis functions, it is easy to combine them linearly and obtain a basis for force-free movement. This is what Figure 6 
shows, where the basis functions $\overline{\boldsymbol{w}}_{i}=\overline{\boldsymbol{u}}_{i}+\mathbb{V}_{i} \boldsymbol{e}$ are plotted, together with their Dirichlet to Neumann map. Notice that, by construction, the integral of the forces on the configuration $\Gamma$ yields zero to machine precision.

Minimizing the expended energy and forcing the displacement to be a given datum, we obtain the same qualitative results as in Ref. [5] and Ref. [6]. The approach we propose in this paper, however, allows us to study also the problem of finding the optimal stroke without assigning an initial (and final) shape.

The closed paths in shape space for target displacements of $.01 \mathrm{~mm}$ and $.001 \mathrm{~mm}$ can be seen in Figure 7 for both the case where the initial shape is fixed to be $(.2 \mathrm{~mm}, .2 \mathrm{~mm})$ and for the case where no constraints are imposed.
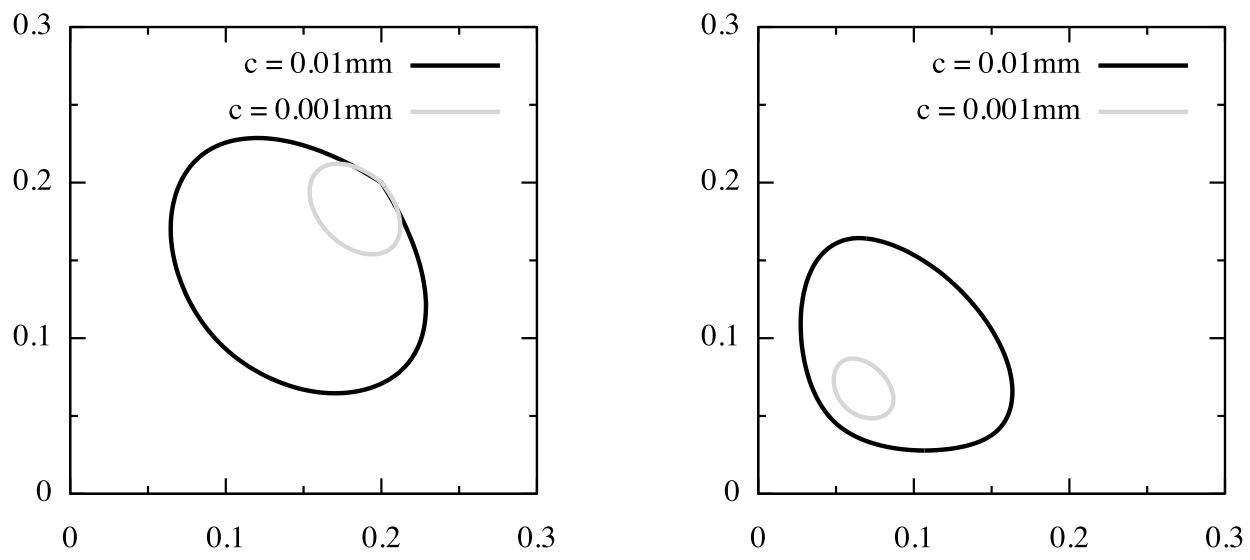

Figure 7: Path in shape space describing optimal strokes for three sphere swimmer of radius $.05 \mathrm{~mm}$, swimming $.01 \mathrm{~mm}$ and $.001 \mathrm{~mm}$ in 1 second, imposing the initial shape $\xi=(.3 \mathrm{~mm}, .3 \mathrm{~mm})$ on the left, and without imposing an initial shape on the right.

In Figure 8 we can observe how the optimal stroke can be separated into a propulsive part and a recovery part, where the propulsive part is the one where the velocity is bigger and positive, while the recovery part is the one where the velocity is smaller and negative.

We would like to emphasize here that, in general, if we fix the initial and final shapes, the velocity is free to be discontinuous at $t=k T$ with $k$ integer, however the truly optimal stroke (the one selected without impositions on 

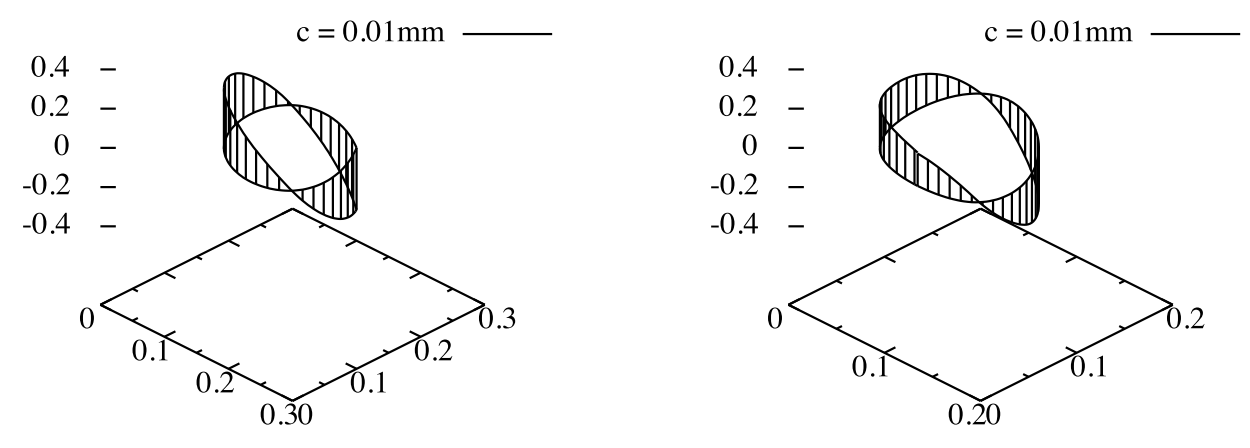

Figure 8: Diagram of translational velocities for three sphere swimmer of radius $.05 \mathrm{~mm}$ swimming $.01 \mathrm{~mm}$ in 1 second imposing the initial shape $\xi=$ $(.3 \mathrm{~mm}, .3 \mathrm{~mm})$ on the left, and without imposing an initial shape on the right.

the starting shape) is one where also the velocity is continuous, as shown in Figure 7 on the right.

\subsection{Stick and Donut swimmer}

We now present a new model swimmer, which simulates the swimming mechanism of many biological organisms made of a body and a propulsive apparatus consisting of appendages that change shape to induce propulsion. A nice example is Chlamydomonas Reinhardtii, a unicellular eukaryotic alga with a body size of roughly $10 \mu \mathrm{m}$, that swims by executing a movement with its two flexible flagella which is closely reminiscent of the breast stroke of an olympic swimmer. An axisymmetric version of this swimming style, though at larger scales, is that of the jelly fish. In the model swimmer we propose, the body is schematized with a cylinder capped with two half-spheres at the ends (the Stick) and the propulsive apparatus is schematized with a torus with elliptical cross-section of variable major and minor radii (the Donut).

Figure 9 shows a section of the swimmer on the $(x, \sigma)$-half plane, for $\theta=0$. The radius of the stick is set to be $R_{0}$, and all other dimensions are scaled with respect to this one, so that the touching distance between the 
stick and the donut is fixed to $3 R_{0} / 2$ and the volumes of the stick and the donut are fixed and equal.

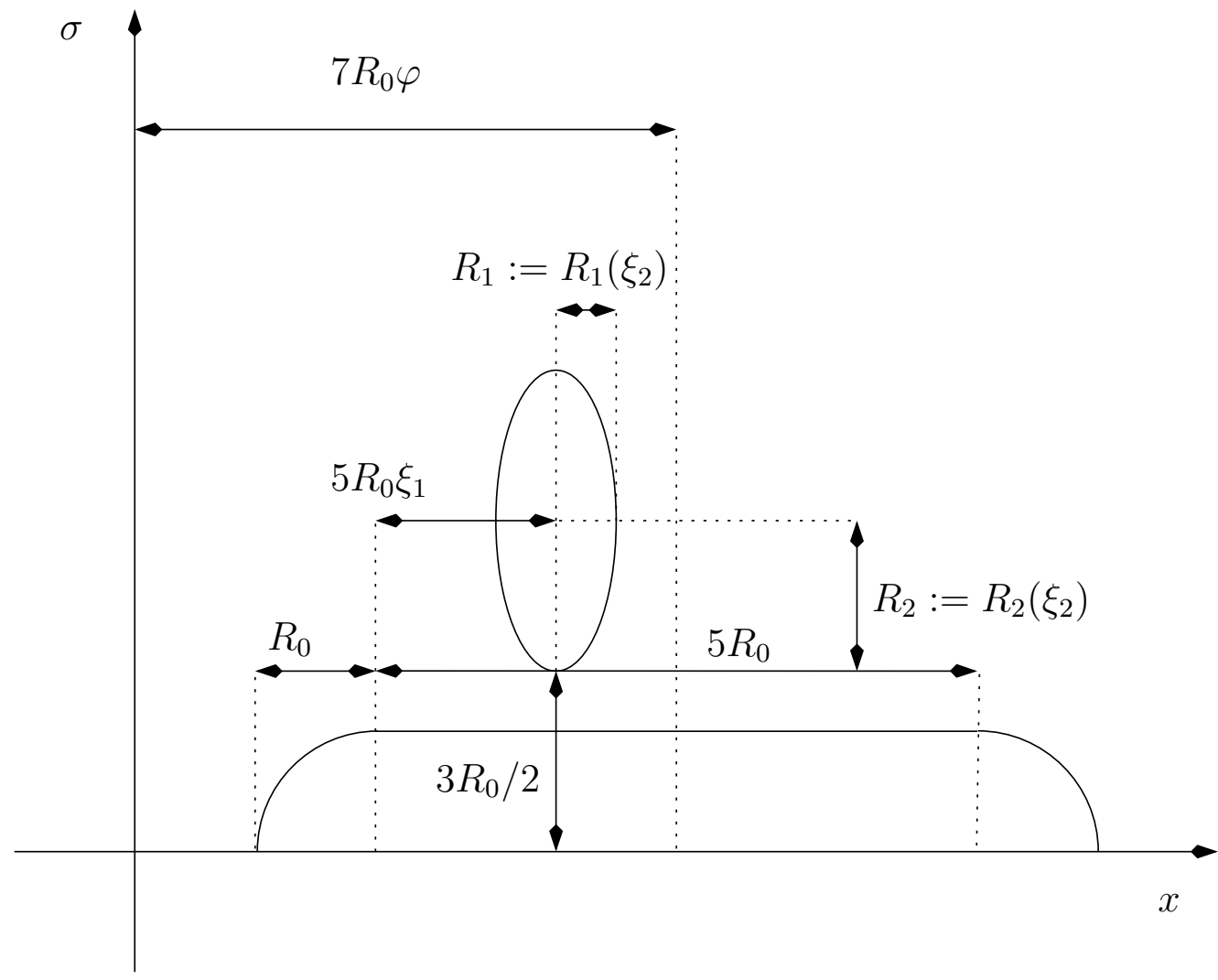

Figure 9: Definition of the "Stick and Donut" swimmer.

Swimming is achieved by translating the center of the donut along the direction of the stick (non-dimensional shape variable $\xi_{1}$, constrained in the interval $[0,1])$, so that the center of the donut is always inside the "body" of the swimmer), and by varying the radii of the donut section.

For the non-dimensional shape parameter $\xi_{2}=0$, the horizontal radius $R_{1}$ of the donut section is equal to $5 R_{0} / 2$, i.e., it has the same length of the stick without the half spheres, while for $\xi_{2}=1$, the horizontal radius $R_{1}$ is equal to $R_{0} / 5$.

The vertical radius of the swimmer and the vertical position of the center 
of the ellipse section are adjusted automatically as a function of $\xi_{2}$ in order to maintain the volume of the donut constantly equal to the volume of the stick and to maintain the distance between the donut and the stick constant.

The absolute position of the swimmer on the axis of symmetry $x$, is given by the non-dimensional variable $\varphi$, so that when $\varphi$ is equal to one, then the swimmer has moved of one entire body length $\left(7 R_{0}\right)$.


Figure 10: Basis functions $\overline{\boldsymbol{u}}_{i}(s)$ and $\boldsymbol{e}(s)$ (left) and their Dirichlet to Neumann map $\boldsymbol{f}_{1}(s)$ and $\boldsymbol{f}_{\boldsymbol{e}}$ (rigth) for $\xi=(.5, .5)$.

Figures 10 and 11 show the basis functions $\overline{\boldsymbol{u}}_{i}$ and $7 R_{0} \boldsymbol{e}$ and the corresponding force-free basis functions $\overline{\boldsymbol{w}}_{i}$ with their Dirichlet to Neumann maps. From Figure 11 it is evident how changes in the shape parameter $\xi_{2}$ do not induce much displacement along the axis of symmetry.

We constrain the swimmer shape variables to be included in the nondimensional box $[0,1]^{2}$, whose corners are shown in Figure 12 , A square stroke that explores in a clockwise manner this shape space is presented in 

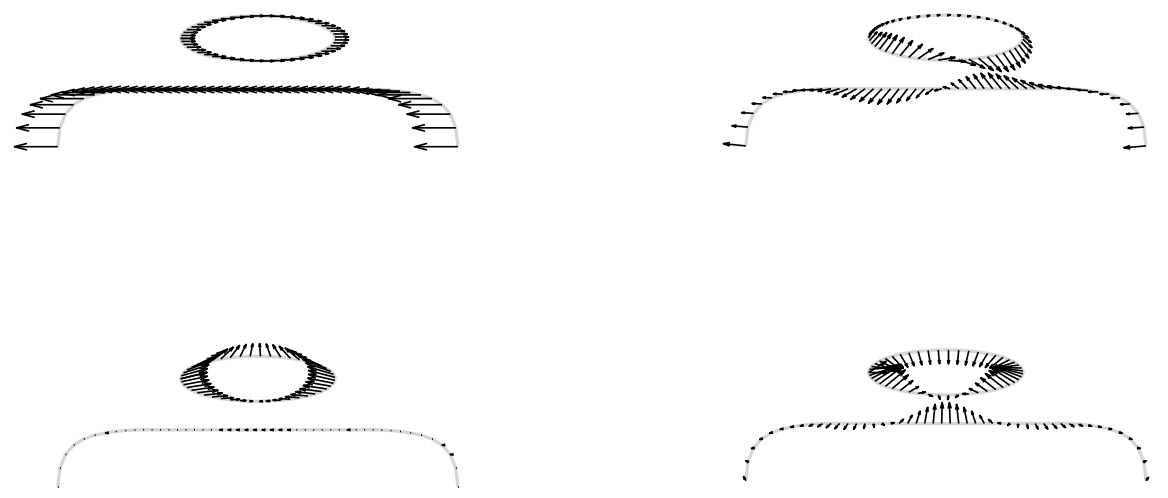

Figure 11: Force free basis functions $\overline{\boldsymbol{w}}_{i}(s)$ (left) and their Dirichlet to Neumann map $\boldsymbol{f}_{\overline{\boldsymbol{w}}_{i}}$ (rigth) for $\xi=(.5, .5)$.

Figure 13 .

The fact that changes in the shape parameter $\xi_{2}$ have little effect on the overall displacement of the swimmer can also be inferred from the left and right sides of the square stroke, in the right part of Figure 13 , which shows how the velocity $\dot{\varphi}$ of the swimmer due to changes in $\xi_{2}$ are negligible when compared with the velocity due to changes in $\xi_{1}$ (top and bottom sides of the square stroke).

The separation of stroke cycles into a power or propulsive phase, in which appendages have maximal hydrodynamical resistance, and a recovery phase, in which the swimmer tries to minimize viscous drag forces on its propulsive appendages, are very common in nature. A typical example, schematically depicted in Figure 14, is the ciliary stroke cycle.

Our model swimmer mimics this behavior by alternating propulsive phases (top side of the square stroke in Figure 13 , i.e., $\xi_{1}$ varying from zero to one while $\xi_{2}$ is close to one) with a recovery phases (bottom side of the square stroke in Figure 13 , i.e., $\xi_{1}$ varying from one to zero while $\xi_{2}$ is close to zero).

In order to compare the stick and donut swimmer with the three sphere swimmer, we set the radius $R_{0}$ of the swimmer such that the total volume of the stick and donut swimmer is equal to the volume of the three sphere swimmer with radius .05 . This gives a radius $R_{0}$ of approximately $.034 \mathrm{~mm}$. 

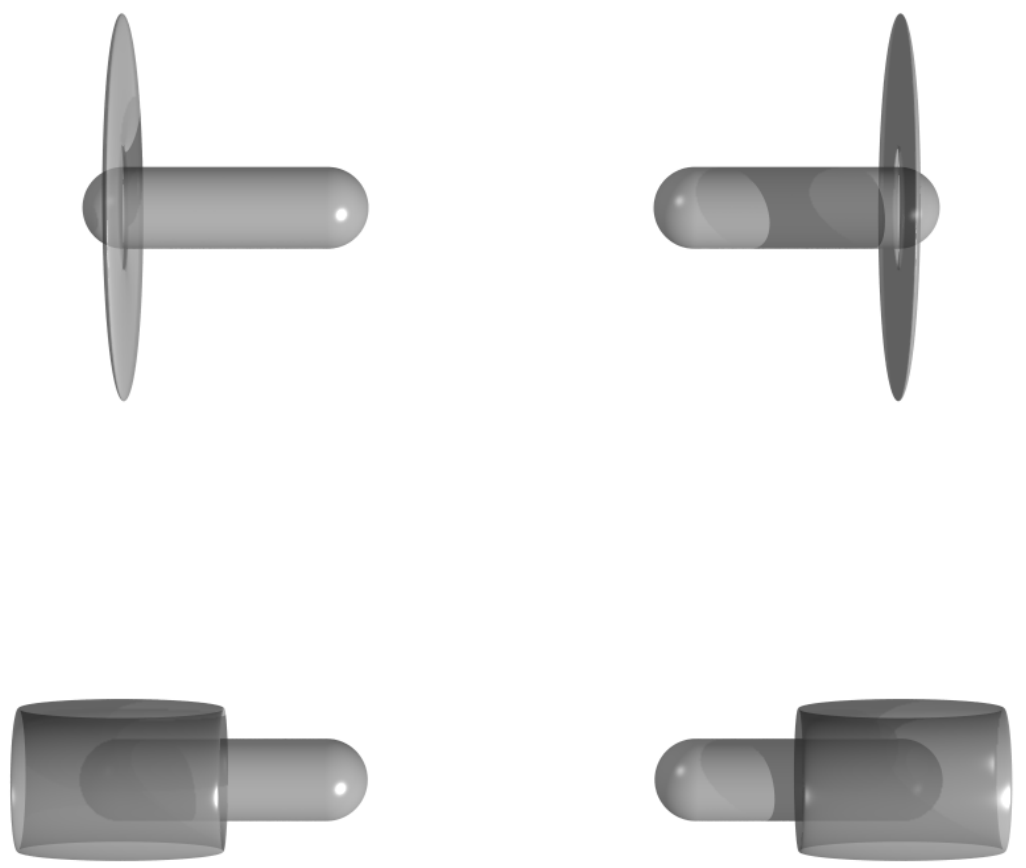

Figure 12: Extremal shape configurations for the stick and donut swimmer: from left to right and from bottom to top: $\xi=(0,0), \xi=(1,0), \xi=(0,1)$ and $\xi=(1,1)$.

Figure 15 shows two optimal strokes found with our method which lead to displacements in 1s of $.01 \mathrm{~mm}$ and $.001 \mathrm{~mm}$. In the first case, the energy consumption is around $.126 \mathrm{pJ}$, while in the second case it is $.010 \mathrm{pJ}$. This should be compared with the energy consumed by an "equivalent" three sphere swimmer, namely, a swimmer with the same volume as this one, swimming at the same average velocity. The three sphere swimmer energy consumption to swim $.01 \mathrm{~mm}$ in one second is $.183 \mathrm{pJ}$, while its energy consumption to swim $.001 \mathrm{~mm}$ in one second is .018pJ.

In the long distance, the stick and donut swimmer is about $45 \%$ more efficient than the optimal three sphere swimmer, while for the shorter distance, the increase in efficiency is about $75 \%$.

A collection of animations referring to the optimal strokes of the swim- 

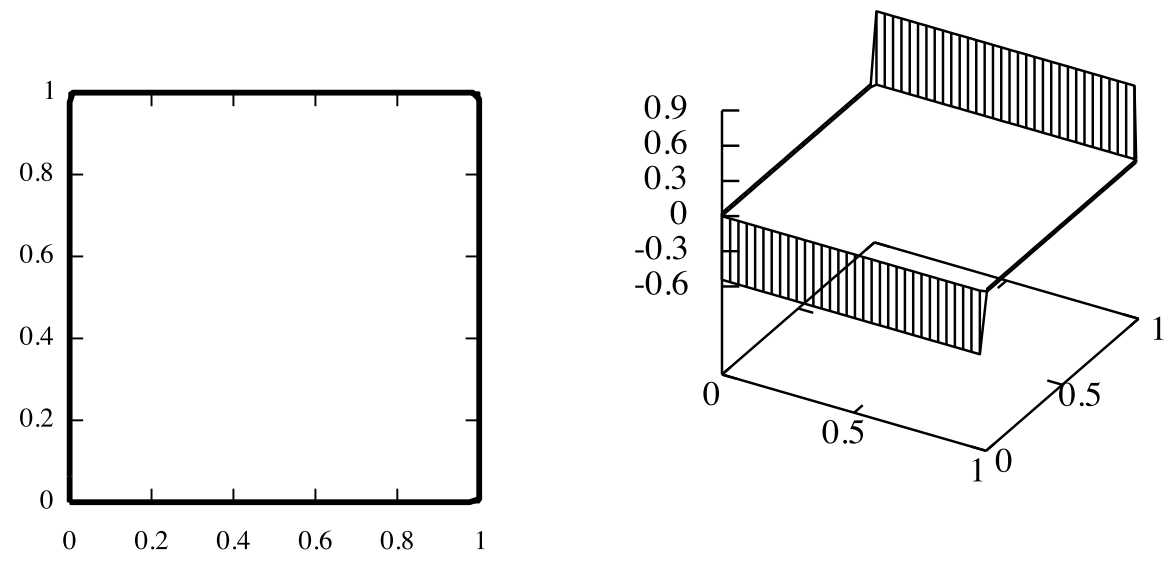

Figure 13: Path in shape space describing a square strokes for stick and donut swimmer with base radius of $.034 \mathrm{~mm}$, swimming $.034 \mathrm{~mm}$ in 1 second (left), with its propulsion diagram (right).

mers presented in this work (Figures 7 and 15) can be viewed on-line on the home page of the corresponding author. [17]

\section{Acknowledgement}

The first author benefited from the support of the "Chair Mathematical modelling and numerical simulation, F-EADS - Ecole Polytechnique - INRIA $-\mathrm{F}-\mathrm{X} "$.

\section{References}

[1] E. Purcell, Life at low Reynolds number, Am. J. Phys 45 (1) (1977) $3-11$.

[2] F. Alouges, A. DeSimone, A. Lefebvre, Biological fluid dynamics, nonlinear partial differential equations, In: Springer Encyclopedia of Complexity and Systems Science. 


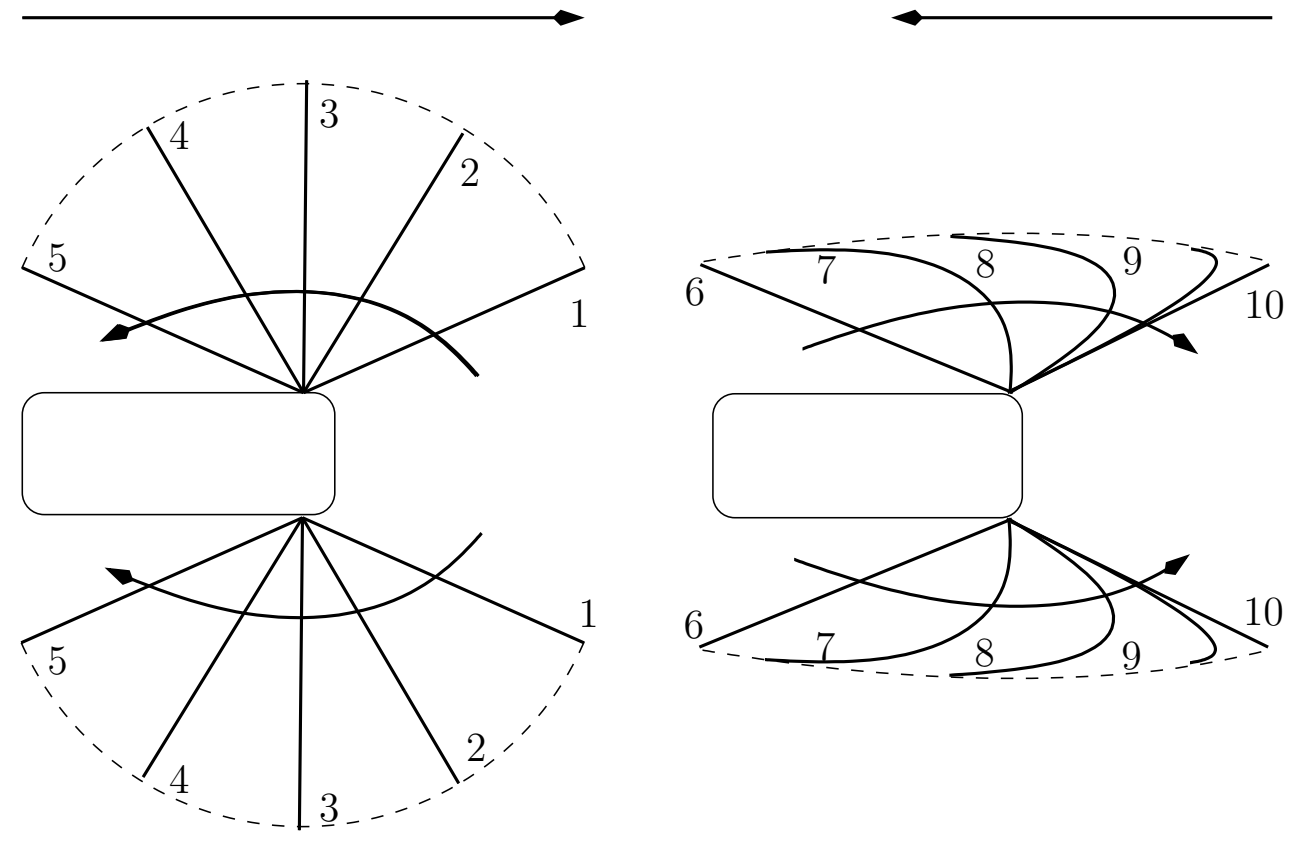

Figure 14: Ciliary stroke cycle. Power phase (left) and recovery phase (right).

[3] A. Najafi, R. Golestanian, Simple swimmer at low Reynolds number: Three linked spheres, Phys. Rev. E 69 (6) (2004) 062901.

[4] J. E. Avron, O. Gat, O. Kenneth, Optimal swimming at low Reynolds numbers, Phys. Rev. Lett. 93 (18) (2004) 186001.

[5] F. Alouges, A. DeSimone, A. Lefebvre, Optimal strokes for low Reynolds number swimmers: an example, J. Nonlinear Sci. 18 (3) (2008) 277-302.

[6] F. Alouges, A. DeSimone, A. Lefebvre, Optimal strokes for axisymmetric microswimmers, The European Physical Journal E 28 (3) (2009) 279284.

[7] R. Dautray, J.-L. Lions, Mathematical analysis and numerical methods for science and technology, Springer, 2000.

[8] C. Pozrikidis, Boundary integral and singularity methods for linearized viscous flow, Cambridge Texts in Applied Mathematics, Cambridge University Press, Cambridge, 1992. 

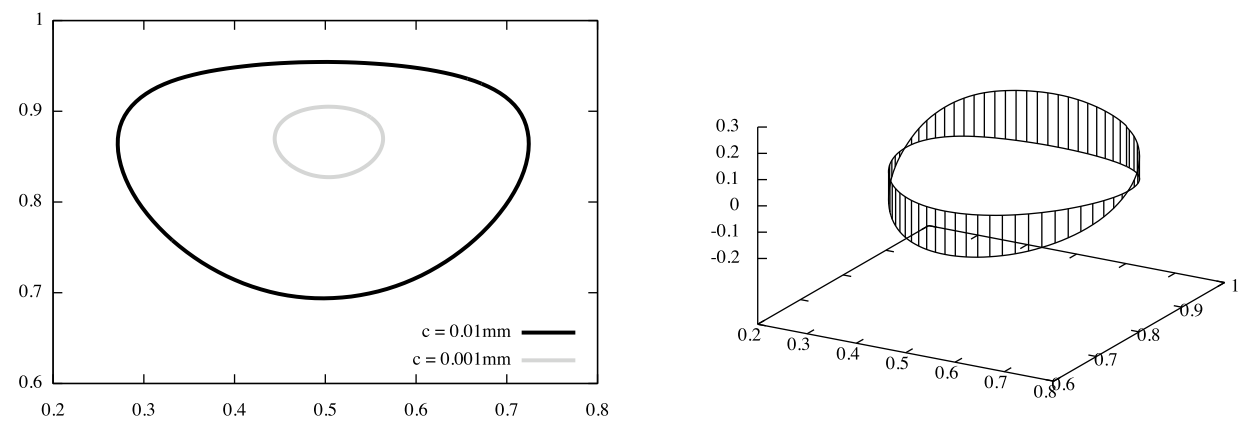

Figure 15: Path in shape space describing optimal strokes for stick and donut swimmer with base radius of $.034 \mathrm{~mm}$, swimming $.01 \mathrm{~mm}$ and $.001 \mathrm{~mm}$ in 1 second, without imposing initial shape (left) and propulsion diagram for the bigger stroke (right).

[9] W. Bangerth, R. Hartmann, G. Kanschat, deal.ii-a general-purpose object-oriented finite element library, ACM Trans. Math. Softw. 33 (4) (2007) 24.

[10] W. Bangerth, R. Hartmann, G. Kanschat, deal. II Differential Equations Analysis Library, Technical Reference.

URL http://www.dealii.org

[11] J. Nocedal, S. J. Wright, Numerical Optimization, Springer, 1999.

[12] M. A. Heroux, R. A. Bartlett, V. E. Howle, R. J. Hoekstra, J. J. Hu, T. G. Kolda, R. B. Lehoucq, K. R. Long, R. P. Pawlowski, E. T. Phipps, A. G. Salinger, H. K. Thornquist, R. S. Tuminaro, J. M. Willenbring, A. Williams, K. S. Stanley, An overview of the Trilinos project, ACM Trans. Math. Softw. 31 (3) (2005) 397-423.

[13] L. Piegl, W. Tiller, The NURBS book (2nd ed.), Springer-Verlag New York, Inc., New York, NY, USA, 1997. 
[14] C. Pozrikidis, A practical guide to boundary element methods with the software library BEMLIB, Chapman \& Hall/CRC, Boca Raton, FL, 2002.

[15] J. Roumeliotis, A Boundary Integral Method applied to Stokes Flow, Ph.D. thesis, School of Mathematics and Statistics, UNSW, Australia (2000).

[16] R. A. Bartlett, Mathematical and high-level overview of moocho, Tech. Rep. SAND2009-3969, Sandia National Laboratories (2009).

[17] L. Heltai, Optimal Swimmers. URL http://people.sissa.it/ heltai/swimmers.php 